\title{
Oligomerization of Chromium(III) and its Impact on the Oxidation of Chromium(III) by Hydrogen Peroxide in Alkaline Solutions
}

\author{
Linfeng Rao, ${ }^{* a}$ Zhicheng Zhang, ${ }^{\text {a Judah I. Friese, }}{ }^{\text {b Ben Ritherdon, }{ }^{\text {b }} \text { Sue B. Clark, }}$ \\ Nancy J. Hess, ${ }^{\mathrm{c}}$ Dhanpat Raic \\ ${ }^{a}$ Lawrence Berkeley National Laboratory, Berkeley, CA 94720, USA. \\ E-mail: LRao@lbl.gov; Fax: 510-486-5596 \\ ${ }^{b}$ Washington State University, Pullman, WA 99164, USA \\ ${ }^{c}$ Pacific Northwest National Laboratory, Richland, WA 99352, USA
}

\section{Graphical Contents Entry}

Kinetic studies on the oxidation of separated/characterized chromium(III) oligomers by hydrogen peroxide indicate that the degree of oligomerization has a significant impact on the rate of oxidation in alkaline solutions. The oxidation rate constant decreases in the order: monomer $>$ dimer $>$ trimer $>$ aged/unseparated chromium(III) solution where higher oligomers dominate.

\section{Summary}

Monomeric, dimeric and trimeric chromium(III) species in solution were separated by ion exchange and characterized with UV/Vis absorption and Extended X-ray Absorption Fine Structure Spectroscopy (EXAFS). The kinetics of the oxidation of the separated species by hydrogen peroxide in alkaline solutions was studied by conventional and stopped-flow $\mathrm{UV} / \mathrm{Vis}$ absorption spectroscopy. Results indicate that the intensity of $\mathrm{Cr}-\mathrm{Cr}$ scattering in the EXAFS spectra $\left(d_{\mathrm{Cr}-\mathrm{Cr}} \sim 2.99 \AA\right)$, a measure of the degree of oligomerization, increases as the solution alkalinity is increased. As the oligomerization proceeds, the rate of oxidation by hydrogen peroxide in alkaline solutions decreases in the order: monomer $>$ dimer $>$ trimer $>$ aged/unseparated alkaline chromium(III) solution where higher 
oligomers dominate. The dominant redox pathway has an inverse order with respect to $C_{\mathrm{NaOH}}$. The data suggest that the rate-determining step involves the weakening of the bridging bonds in the oligomer and a concomitant release of one hydroxyl group from the chromium(III) moiety upon the attack by hydrogen peroxide.

\section{Introduction}

Safe disposal of high-level nuclear wastes (HLW) requires advanced technologies including vitrification of HLW into glass to immobilize the radioactive materials. However, chromium, one of the non-radioactive components in the HLW sludge, has raised particular concern because it forms and settles as a refractory spinel-type crystalline phase in the glass melter, adversely affecting the vitrification process. Consequently, it is necessary to remove chromium from the waste sludge before vitrification. $^{1}$

The chromium in the sludge is mainly chromium(III) and the majority of the HLW is under alkaline conditions. Thus, to develop efficient strategies for the removal of chromium from the sludge, it is important to understand the chemical behavior of chromium(III) in alkaline solutions. However, most of the studies on chromium(III) have been conducted in acidic to neutral systems, ${ }^{2-13}$ while few data exist for alkaline solutions. For example, information is available on the hydrolysis and oligomerization of chromium(III) in acidic to neutral solutions. The structures of a few oligomers (dimer, trimer and tetramer) in solution were postulated ${ }^{10-13}$ based on a variety of techniques including $\mathrm{pH}$ titrations, ${ }^{11}{ }^{18} \mathrm{O}$ exchange, ${ }^{12}$ freezing point lowering, ${ }^{13}$ magnetic susceptibility measurements and EPR. ${ }^{13}$ The structures of these oligomers in crystals have also been elucidated by X-ray crystallography. ${ }^{14,15}$ On the contrary, information on 
alkaline solutions is scarce and uncertain. It was generally accepted that anionic monomers, $\mathrm{Cr}(\mathrm{OH})_{\mathrm{j}}{ }^{(\mathrm{j}-3)-}(\mathrm{j}=4,5$ or 6$)$, form in alkaline solutions and become dominant when $\mathrm{pH}$ is above $12 .^{2,16}$ However, this assumption was solely based on the fitting of solubility data and the species have not been identified. In fact, recent studies ${ }^{17}$ have cast doubt on the formation of $\mathrm{Cr}(\mathrm{OH})_{4}{ }^{-}$and the speciation of chromium(III) in alkaline solutions still remains uncertain. This is not surprising because these systems are extremely complex and characterization techniques directly applicable to alkaline solutions are not always available. It should be pointed out that, even for the acidic systems, there is discrepancy between the structures of the trimer and tetramer in the crystal and those postulated in the solution (Fig. 1), suggesting more characterization for the solution species is needed.

In recent years, Extended X-ray Absorption Fine Structure (EXAFS) has been shown to be a useful technique that can probe the neighboring atoms of chromium in solution and on minerals. The structures of hydrated chromium(III) ions in acidic solutions ${ }^{18,19}$ and chromium(III) sorbed on bentonite ${ }^{20}$ and oxide-water interface ${ }^{21}$ have been elucidated. However, this technique has not been used to directly study the chromium(III) speciation in alkaline solutions. Therefore, one objective of the present work is to extend the study of the oligomerization of chromium(III) from acidic solutions to strongly alkaline solutions by EXAFS. Additional structural information on chromium(III) oligomers in solution is obtained and helps to understand the impact of oligomerization on the chemical behavior of chromium(III).

One of the strategies proposed for the removal of chromium from the sludge is oxidative dissolution, using oxidants including oxygen, ozone, permanganate, ferrate and 
hydrogen peroxide. This requires a thorough understanding of the redox reactions between chromium(III) and the oxidants in alkaline solutions. Unfortunately, such information is extremely scarce. A few studies have been conducted on the oxidation of chromium(III) in nuclear waste sludges by oxygen, ozone, permanganate, ${ }^{22}$ and ferrate. ${ }^{23}$ The results are useful, to some extent, to the development of sludge washing strategies. However, they are mostly phenomenological and do not reveal how the speciation of chromium(III) in alkaline solutions, oligomerization in particular, would affect the oxidation. Therefore, the second objective of this work is to study the oxidation of separated and characterized chromium(III) oligomers so that the impact of oligomerization on the oxidation is evaluated. Hydrogen peroxide was studied as the oxidant because it possesses appropriate redox potential in alkaline solutions and doesn't generate secondary solid wastes to increase the waste volume. Besides, no mechanistic studies have been reported in the literature on the oxidation of chromium(III) by hydrogen peroxide in alkaline solutions.

\section{Results And Discussion}

\subsection{Distribution of oligomers obtained by ion-exchange}

The distribution of oligomers in the five aged solutions with different $\mathrm{pH}$ (see Experimental Section) is shown in Fig. 2. When $\mathrm{pH}$ is lower than 2.7, monomeric chromium(III) cations are the dominant species and oligomerization is negligible. The dimeric and trimeric chromium(III) species range from $0 \%$ to $10 \%$ in the $\mathrm{pH}$ region from 2.6 to 3.5. The percentage of higher oligomers increases rapidly with the increase in $\mathrm{pH}$. If a tetramer $\left(\mathrm{Cr}_{4}(\mathrm{OH})_{6}{ }^{6+}\right)$ is assumed to dominate in the fraction of higher oligomers, the concentration quotients $\left(Q_{\mathrm{pq}}\right)$ for the reaction 


$$
p \mathrm{Cr}^{3+}+q \mathrm{H}_{2} \mathrm{O} \leftrightarrows \mathrm{Cr}_{\mathrm{p}}(\mathrm{OH})_{\mathrm{q}}^{(3 \mathrm{p}-\mathrm{q})+}+q \mathrm{H}^{+}
$$

can be estimated from these data. The values are listed in Table 1 in comparison with earlier values from the literature. The $\log Q_{22}$ value agrees well with those in the literature. $^{10,11,13}$ The $\log Q_{46}$ value is also consistent with that from Ref.11. However, there is greater discrepancy between our $\log Q_{34}$ value and those from previous studies. Our value suggests that the tendency of forming the trimer in solution is much weaker than predicted by the previous values, demonstrated by the fact that the amount of trimer never exceeds a few percent of the total chromium(III) in the solutions with a $\mathrm{pH}$ range from 2.6 to 3.5 (Fig. 2).

\subsection{Oligomerization studied by EXAFS}

2.2.1 Characterization of separated oligomers. The edge position of the chromium kedge absorption spectra and the absence of a strong pre-edge feature due to the $3 \mathrm{~d}-4 \mathrm{p}$ mixing in the tetrahedral chromate indicate that the oxidation state of chromium was +3 in all the samples. As a result, the analysis of the X-ray absorption spectra was focused on the EXAFS.

The Fourier Transform of the EXAFS spectra for the separated oligomers (in solution and on the resin) is shown in Fig. S1, Supplementary Materials. Two single scattering (SS) paths, $\mathrm{Cr}-\mathrm{O}$ at $\sim 1.97 \AA$ and $\mathrm{Cr}-\mathrm{Cr}$ at $\sim 2.98 \AA$, were used in the fitting of the EXAFS data. Multiple scattering (MS) paths involving the cis- Cr-O-O (3.2 - $3.4 \AA)$ and the trans- O-Cr-O (3.93 - $3.96 \AA)$ interactions were also tested. Though the inclusion of MS paths slightly improved the fit around $3.4 \AA$, it was found to have insignificant 
influence on the best fit parameters (this effect is further discussed in section 2.2.2). The fitting results are summarized in Table 2. For the monomer and dimer, the results for the solution and the resin samples are in good agreement. However, for the trimer, only the results for the solution sample were obtained because the spectra for the resin sample were in poor quality.

A good fit was obtained for the monomer with one oxygen shell (six oxygen atoms) at $1.97 \AA$, consistent with the results in the literature (six oxygen atoms at $1.966 \pm 0.008$ $\AA$ ) obtained by EXAFS and LAXS (Large Angle X-ray Scattering). ${ }^{18}$ For the dimer, in addition to the oxygen shell at 1.94-1.96 $\AA$ ( 6 oxygen atoms), the best fit was obtained with a chromium shell at 2.98-2.99 $\AA\left(N_{\mathrm{Cr}-\mathrm{Cr}}=0.8-1.1\right)$, in good agreement with the crystal structure. ${ }^{14,15}$ However, it should be pointed out that the crystal structure of the dimer (Fig. 1a) shows a shorter bridging $\mathrm{Cr}-\mathrm{O}$ distance (1.936 $\AA$ ) than the terminal $\mathrm{Cr}-$ $\mathrm{OH}_{2}(1.988 \AA),{ }^{14}$ but our EXAFS data show only a single oxygen shell at $1.94 \AA-1.96 \AA$ (Table 2). This discrepancy suggests that, either the small difference in bond distance is not resolved in our EXAFS measurements or the structure of dimer in solution is less rigid and indeed slightly different from that in the crystal due to the effect of solvation.

For the trimer, the best fit was obtained with six oxygen atoms at $1.94 \AA$ and two chromium atoms at $2.98 \AA\left(N_{\mathrm{Cr}-\mathrm{Cr}}=1.9 \pm 0.5\right.$, Table 2$)$. This is consistent with the triangular structure postulated for the solution, where each chromium has two neighboring chromium atoms at an equal distance (Fig. 1c). Even with the uncertainty of \pm 0.5 in $N_{\mathrm{Cr}-\mathrm{Cr}}$, our data do not seem to support the postulated linear structure for the trimer in the solution (Fig. 1b) because the average $N_{\mathrm{Cr}-\mathrm{Cr}}$ at $2.98 \AA$ in the linear configuration should be 1.33. Moreover, our data do not agree with the crystal structure 
of trimer, in which the $\mathrm{Cr}-\mathrm{Cr}$ distances are 2.86, 3.50 and $3.56 \AA$, respectively (Fig. 1d). We think the configuration (c) in Fig. 1 probably better represents the structure of trimer in solution because it is consistent with the $\mathrm{pH}$ titration data ${ }^{11}$ and our EXAFS measurements - both being conducted in solution. It is interesting to note that recent results obtained by Capillary Electrophoresis (CE), a technique capable of differentiating species in solution by charge density, also favor the triangular configuration over the linear configuration for the chromium(III) trimer in solution. ${ }^{24}$

The data in Table 2 show that the number of $\mathrm{Cr}$-Cr scattering path $\left(N_{\mathrm{Cr}-\mathrm{Cr}}\right)$ increases in the order: monomer $(0)<\operatorname{dimer}(0.8 \pm 0.5)<\operatorname{trimer}(1.9 \pm 0.5)$, indicating that EXAFS is a valuable technique to probe the oligomerization of chromium(III). For the first time, these data have confirmed the nature of the individual oligomers of chromium(III) separated by ion exchange and, in a sense, calibrated the EXAFS when applied to characterize unknown chromium(III) species in alkaline solutions.

2.2.2 Effect of alkalinity on oligomerization. The EXAFS spectra and the Fourier transforms of the spectra for unseparated chromium(III) solutions with different alkalinity are shown in Fig. 3. In general, good fits were obtained by using only the two SS paths ( $\mathrm{Cr}-\mathrm{O}$ at $\sim 1.97 \AA$ and $\mathrm{Cr}-\mathrm{Cr}$ at $\sim 2.98 \AA$ ), but the minor peak at $\sim 3.4 \AA$ seemed not to be accounted for adequately (Fig. 3b). The weak features up to about $4 \AA$ could be originated from either the MS within the first Cr-O shell or the SS from a more distant $\mathrm{Cr}-\mathrm{Cr}$ shell beyond $3 \AA$ (as in higher oligomers), ${ }^{21}$ or both. There have been arguments in the literature ${ }^{18,19}$ as to whether the MS within the first coordination shell or the SS from more distant shell(s) is of greater importance in interpreting the EXAFS data. However, since there are no $\mathrm{Cr}-\mathrm{Cr}$ interactions in the monomer and the minor peak at $3.4 \AA$ still 
shows in the Fourier Transform of its EXAFS, it is reasonable to assume that, in these systems, the MS within the first $\mathrm{Cr}-\mathrm{O}$ shell is more important than the SS from a more distant $\mathrm{Cr}-\mathrm{Cr}$ shell. To test this assumption, fitting with several combinations of different scattering paths (SS and MS) were performed and the goodness of the fits were compared. We found that, indeed, inclusion of a Cr-Cr SS path around $4 \AA$ resulted in poor fits for the oligomer systems so this path was not included in subsequent fittings. As to the MS paths, calculations with the program $\mathrm{FEFF}^{25}$ indicated that the cis- $\mathrm{Cr}-\mathrm{O}-\mathrm{O}$ (3.2 - 3.4 $\AA$ ) MS interactions could be ignored (only 6-8\% contribution with respective to $100 \%$ for the Cr-O SS at $1.97 \AA$ ), but the MS interactions at $\sim 3.90 \AA$ originating from the 4-legged trans- O-Cr-O path could have some effect ( $\sim 35 \%$ contribution). As a result, the latter was included in the fits. Rather than varied independently, this path was linked directly to the value of the coordination number $(N)$ of the first Cr-O shell. ${ }^{26}$ Slight improvement was obtained in the refinements for the minor feature at about $3.4 \AA$, but the fitting parameters for the first $\mathrm{Cr}-\mathrm{O}$ and $\mathrm{Cr}-\mathrm{Cr}$ shells were essentially unchanged, compared to the fits with only the two SS paths.

The fitting parameters are listed in Table 2. As shown in Table 2, the values of $N_{\mathrm{Cr}-\mathrm{Cr}}$ (at $\sim 2.99 \AA$ ) increase as the alkalinity of the solutions is increased. In concentrated sodium hydroxide $\left(5.5 \mathrm{~mol} \mathrm{dm}^{-3}\right.$ to $7.8 \mathrm{~mol} \mathrm{dm}^{-3}$ ), the $N_{\mathrm{Cr}-\mathrm{Cr}}$ ranges from 2.3 to 2.7 , which clearly shows that chromium(III) exists as oligomers in alkaline solutions with an average degree of oligomerization higher than that of trimer. This conclusion is contradictory to the earlier belief that oligomerization of chromium(III) is only important in weakly acidic to neutral solutions ( $\mathrm{pH} 3 \sim 8$ ) and monomeric $\mathrm{Cr}(\mathrm{OH})_{4}{ }^{-}$dominates when $\mathrm{pH}$ is above $12 .{ }^{2,16}$ Due to the unavailability of characterization techniques such as EXAFS that can 
directly probe the oligomerization in alkaline solutions, the speciation in the earlier studies was based on the fitting of solubility curves and, evidently, needs to be reevaluated. Our EXAFS data clearly demonstrate that chromium(III) oligomers are important in alkaline solutions and must be included in the interpretation of solubility and redox data. Recently, the impact of oligomerization on the solubility of chromium(III) hydroxide in alkaline solutions has been discussed in another study. ${ }^{27}$ The present study is focused on the impact of oligomerization on the oxidation of chromium(III) in alkaline solutions.

\subsection{Oxidation of chromium(III) by hydrogen peroxide in alkaline solutions}

2.3.1 Rate equations and overall reactions. The rate constants, $k, k^{\prime}, k_{\mathrm{obs}}$ and $k_{\mathrm{OH}}$, are defined in Experimental Section. Detailed kinetic data are summarized in Table S1, Supplementary Materials.

The reaction order with respect to chromium(III). Under pseudo-first-order conditions with respect to the concentration of chromium(III), plots of $\ln \left(A_{\infty}-A_{\mathrm{t}}\right)$ vs. time are linear, suggesting a first-order reaction with respect to $\left[\mathrm{Cr}_{n}(\mathrm{III})\right]($ where $\mathrm{n}=1,2$ and 3 for the monomer, dimer and trimer). From these plots, the values of $k_{\text {obs }}$ were calculated (Table S1, Supplemental Materials).

The earlier experiments under the pseudo-first-order conditions by conventional spectroscopy covered only a narrow range of the concentrations of chromium (e.g., $6.8 \times$ $10^{-5}$ to $9.7 \times 10^{-5} \mathrm{~mol} \mathrm{dm}^{-3}$ for the monomer). As one anonymous reviewer correctly pointed out, this may raise doubts about the validity of the reaction order established for the chromium species, the chromium(III) monomer in particular, because the latter may 
undergo oligomerization on a comparable time scale while being oxidized. In addition, the oxidation of the monomer was very rapid, implying that the technique of conventional spectroscopy (by hand-mixing) may cause high uncertainties in the results. Therefore, additional experiments on the monomer were conducted with the stopped-flow spectroscopy, covering a wider range of concentration of chromium $\left(1.2 \times 10^{-5}\right.$ to $1.2 \times$ $10^{-4} \mathrm{~mol} \mathrm{dm}^{-3}$ ). Representative "kinetic traces" (the absorbance at $372 \mathrm{~nm}$ vs. time) obtained by stopped-flow spectroscopy were shown in Fig. S2, Supplementary Materials. The initial rates, $\left(\mathrm{d} A_{t} / \mathrm{d} t\right)_{0}$, were calculated from these data. Fig. 4 shows that the plot of $\log \left(\mathrm{d} A_{\mathrm{t}} / \mathrm{d} t\right)_{0}$ vs. $\log C_{\mathrm{Cr}}$ is linear, with a slope of $1.05 \pm 0.11$, supporting previous results that the oxidation of monomer is the first order with respect to chromium.

The reaction order with respect to hydrogen peroxide. At constant $C_{\mathrm{NaOH}}, k_{\mathrm{obs}}$ increases proportionately with the increase of $C_{\mathrm{H} 2 \mathrm{O} 2}$ as shown in Fig. 5, suggesting the reaction is first-order with respect to $C_{\mathrm{H} 2 \mathrm{O} 2}$. The slopes of the lines represent the values of $k_{\mathrm{OH}}$ (Table S1, Supplemental Materials).

The reaction order with respect to hydroxide. The plots of $k_{\mathrm{OH}}$ vs. $C_{\mathrm{NaOH}}{ }^{-1}$ are linear with small intercepts (Fig. 6). Thus, a general rate equation can be expressed as

$$
R=-\mathrm{d}\left[\mathrm{Cr}_{\mathrm{n}}(\mathrm{III})\right] / \mathrm{d} t=k\left[\mathrm{Cr}_{\mathrm{n}}(\mathrm{III})\right]\left[\mathrm{H}_{2} \mathrm{O}_{2}\right]\left(1 /[\mathrm{OH}]+k^{\prime}\right)
$$

The values of $k$ and $k$ ' are calculated from the slopes and intercepts and listed in Table 3. It should be noted that, for the monomer (Fig. 6a), the results from the conventional spectroscopy (solid symbols) and the stopped-flow (open symbols) spectroscopy are in good agreement, confirming the validity of the approach for processing the monomer data. 
Overall reactions. The stoichiometry of the reaction between chromium(III) and hydrogen peroxide was determined to be 2:3 (the molar ratio of the chromium(VI) produced to the hydrogen peroxide consumed), using known amounts of hydrogen peroxide in the presence of excess chromium(III). Taking into consideration that the dominant species of hydrogen peroxide in alkaline solutions is $\mathrm{HO}_{2}^{-}\left(\mathrm{p} K_{\mathrm{a} 1}\right.$ for hydrogen peroxide $\sim 11-12),{ }^{28}$ the overall reactions between the monomer, dimer and trimer of chromium(III) and hydrogen peroxide can be expressed as:

$$
\begin{aligned}
& 2 \mathrm{Cr}(\mathrm{OH})_{4}{ }^{-}+3 \mathrm{HO}_{2}^{-} \rightarrow 2 \mathrm{CrO}_{4}{ }^{2-}+\mathrm{OH}^{-}+5 \mathrm{H}_{2} \mathrm{O} \\
& \mathrm{Cr}_{2} \mathrm{O}_{2}(\mathrm{OH})_{4}{ }^{2-}+3 \mathrm{HO}_{2}^{-} \rightarrow 2 \mathrm{CrO}_{4}{ }^{2-}+\mathrm{OH}^{-}+3 \mathrm{H}_{2} \mathrm{O} \\
& 2 \mathrm{Cr}_{3} \mathrm{O}_{4}(\mathrm{OH})_{4}{ }^{3-}+9 \mathrm{HO}_{2}^{-} \rightarrow 6 \mathrm{CrO}_{4}{ }^{2-}+3 \mathrm{OH}^{-}+7 \mathrm{H}_{2} \mathrm{O}
\end{aligned}
$$

It should be pointed out that our EXAFS data, while providing information on the degree of oligomerization, are insufficient to determine the structure or composition of chromium(III) oligomers in alkaline solutions. Complementary information obtained with other techniques is required. Attempts to prepare crystalline compounds containing oligomers from alkaline solutions have been made but are unsuccessful. Other techniques (e.g., ion exchange, capillary electrophoresis) have been tried to identify the charge and size of the oligomers but the results are inconclusive. As a result, the formula of chromium(III) species in the above equations are postulated based on the structures in acidic solutions (Fig. 1), taking into consideration the likely conversion of hydroxobridges to oxygen bridges. As pointed out by Hall and Eyring, ${ }^{7}$ higher concentrations of hydroxide facilitate the conversion of hydroxo-bridges to oxygen bridges through deprotonation and/or dehydration. By conductometric titrations, they concluded that hydroxo-bridged dimer would change to oxygen-bridged dimer in aged and heated 
chromium(III) solutions and the addition of sodium hydroxide also caused a large increase in the number of oxygen bridges formed. ${ }^{7}$

\subsubsection{Impact of oligomerization on the oxidation and postulated mechanism. As}

shown in Table 3, the oxidation rate constant, $k$, decreases as oligomerization proceeds: Monomer $>$ Dimer $>$ Trimer. Currently, full explanations for the observed order are not possible because they require detailed structural information on the oligomers and the knowledge of the point of attack by hydrogen peroxide. However, this order indicates that higher oligomers are less reactive in the oxidation. Since the reaction product is chromate $\left(\mathrm{CrO}_{4}{ }^{2-}\right)$, the oxidation of chromium(III) oligomers must involve the breaking of hydroxo- or oxygen bridges in the oligomers. The bridging bonds may be weakened when the activation complex between chromium(III) and hydrogen peroxide forms. The order of the rate constant implies that higher activation energy is required to form the activated complex with higher oligomers.

Since the EXAFS data indicate that the average degree of oligomerization in $7.8 \mathrm{~mol}$ $\mathrm{dm}^{-3}$ sodium hydroxide is 2.7 (Table 2), higher than that of trimer, it is interesting to determine the rate of oxidation of this chromium(III) solution and compare with the results from the separated oligomers. To process the kinetic data for this system, we have assumed that one species $\left(\mathrm{Cr}_{\mathrm{n}}(\mathrm{III}), n\right.$ is unknown but definitive) is dominant. ${ }^{*}$ The reaction was found to be the first-order with respect to $\mathrm{Cr}_{\mathrm{n}}(\mathrm{III})$ and there was no need to know the value of $n$ because it is canceled in the calculation. Thus, we could process the

\footnotetext{
${ }^{*}$ It could be a tetramer or a higher oligomer as shown by recent data indicating
} hydrolyzed chromium solutions could form a single polyoxo species probably containing a $\mathrm{Cr}_{12}$ unit. $^{17}$ 
data in the same manner as for the individual oligomers and calculate the apparent constants $k, k^{\prime}, k_{\mathrm{obs}}$ and $k_{\mathrm{OH}}$. Obviously, the oxidation of chromium(III) from this solution occurred even more slowly than the trimer (Table 3). This observation supports the trend that higher oligomers are less reactive in the oxidation by hydrogen peroxide.

The reaction order with respect to hydroxide suggests two parallel reaction pathways (equation 1) - one is inversely dependent on $C_{\mathrm{NaOH}}$ and the other independent of $C_{\mathrm{NaOH}}$. Our data show that the hydroxide-independent pathway is negligible for the monomer and dimer, and insignificant for the trimer and unseparated systems. Thus, the hydroxidedependent pathway is the major pathway for the oxidation of chromium(III) by hydrogen peroxide in alkaline solutions. The inverse dependency of the oxidation rate on $C_{\mathrm{NaOH}}$ means that, either the activated complex between chromium(III) and hydrogen peroxide involves chromium species that are protonated at one of the hydroxy groups or, presumably, the rate-determining step involves the weakening and release of one hydroxyl group from the chromium(III) moiety upon the attack by hydrogen peroxide.

\section{Experimental Section}

\subsection{Chemicals}

All the chemicals were reagent grade or higher and used without further purification. Solutions were prepared with deionized water from a Millipore system. Experiments involving neutral to basic solutions were conducted under nitrogen in a glove box.

Stock solutions of chromium(III) were prepared by dissolving either $\mathrm{CrCl}_{3} \cdot 6 \mathrm{H}_{2} \mathrm{O}$ or $\mathrm{Cr}\left(\mathrm{ClO}_{4}\right)_{3} \cdot 6 \mathrm{H}_{2} \mathrm{O}$ (Aldrich) in water. To remove chloride and any chromium(VI) in the stock solutions, appropriate amounts of sodium hydroxide were added until the $\mathrm{pH}$ was around 9 to precipitate chromium(III) hydroxide. The precipitate was washed $2-3$ times 
with water $(\mathrm{pH} \sim 9)$ and then dissolved with perchloric acid. Solutions of sodium hydroxide were prepared from an oversaturated sodium hydroxide solution and standardized by $\mathrm{pH}$ titration. Stock solutions of hydrogen peroxide were prepared from a solution of 50\% hydrogen peroxide (Aldrich) prior to use and standardized by redox titrations, ${ }^{29}$ using cerium(IV) sulfate (Aldrich). Sephadex SP C-25 cation exchange resin (Aldrich) was used for the separation of chromium(III) species.

All the chromium(III) solutions used in the characterization and the redox studies were filtered prior to use, with the $0.2 \mu \mathrm{m}$ nylon Acrodisc filter (Gelman).

\subsection{Separation of chromium(III) oligomers}

Procedures for separation of chromium(III) oligomers have been described elsewhere. ${ }^{11,30}$ The monomer, dimer and trimer were successively eluted by solutions with increasingly higher ionic strength (from 1 to $4 \mathrm{~mol} \mathrm{dm}^{-3} \mathrm{NaClO}_{4}$ ). Well-separated individual oligomers were obtained by carefully controlling the elution rate and the timing of changing eluting agents. The UV-Vis absorption spectra of the separated solutions (Fig. S3, Supplementary Materials) are identical to those of the monomer, dimer and trimer reported in the literature, ${ }^{11,13,30}$ confirming that good separation was achieved. In our experiment, a fraction of chromium(III) always remained on the top of the column, representing higher chromium(III) oligomers that had very strong affinity to the resin. No tetramer of chromium(III) was separated. The solutions of the separated chromium(III) species were either immediately used for characterization and redox studies or stored in a refrigerator prior to use.

To study the effect of $\mathrm{pH}$ on the oligomerization of chromium(III) in weakly acidic solutions, five chromium(III) solutions of different $\mathrm{pHs}$ were prepared by mixing equal 
volumes of chromium(III) stock $\left(C_{\mathrm{Cr}}=0.1 \mathrm{~mol} \mathrm{dm}^{-3}, C_{\mathrm{HClO} 4} \sim 0.01 \mathrm{~mol} \mathrm{dm}^{-3}, C_{\mathrm{NaClO} 4} \sim 1\right.$

mol $\left.\mathrm{dm}^{-3}\right)$ and a series of sodium hydroxide solutions $\left(C_{\mathrm{NaOH}}=0,0.05,0.1,0.15\right.$ and 0.2

mol dm${ }^{-3}$, respectively). After 62 days, the $\mathrm{pHs}$ of these solutions were steady and measured to be $2.66,2.83,3.00,3.20$ and 3.44 , respectively. Then the ion exchange procedures were followed to separate the chromium(III) species in these solutions. The amounts of individual chromium(III) species were determined by absorption spectrophotometry after being completely oxidized by hydrogen peroxide. The amount of the chromium(III) that remained on the column and represented the chromium(III) with higher affinity to the resin was calculated from the difference between the total amount of chromium(III) in the initial solutions and the sum of the chromium(III) species in all the eluates.

\subsection{EXAFS}

To prepare the solution samples of chromium(III) for the EXAFS experiments, a small aliquot of the solution was placed in between two thin polyethylene sheets (thickness $0.05 \mathrm{~mm}$ ) and thermally sealed to form a thin polyethylene bag with the approximate size of $30 \mathrm{~mm}(\mathrm{~L}) \times 6 \mathrm{~mm}(\mathrm{~W}) \times 0.5 \mathrm{~mm}(\mathrm{D})$. The bags were mounted on an aluminum sample holder with Scotch tape.

Chromium K-edge EXAFS spectra were collected at the Stanford Synchrotron Radiation Laboratory (SSRL) on wiggler beamline 4-1 under normal ring operating conditions $(3.0 \mathrm{GeV}, 50-100 \mathrm{~mA})$. Energy scan of the polychromatic X-ray beam were obtained using a $\mathrm{Si}(220)$ double-crystal monochromator. The vertical slit width was 0.5 $\mathrm{mm}$, which reduces the effects of beam instabilities and monochromator glitches while providing ample photon flux. The higher order harmonic content of the beam was 
reduced by detuning the crystals in the monochromator so that the incident flux was reduced to $50 \%$ of its maximum at the scan ending energy. The samples were measured in the fluorescence mode using either a 13 -element or a 4 -element Ge detector, ${ }^{31}$ to a $k=$ $13 \AA^{-1}$. Energy calibration was based on assigning the first inflection point of the absorption edge of a chromium metal foil standard to $5989 \mathrm{eV}$. The EXAFS spectra were fit with the R-space X-ray Absorption Package (RSXAP), ${ }^{32}$ using parameterized phase and amplitude functions generated by the program $\mathrm{FEFF}^{25}$ or FEFF8 with grimaldite ( $\alpha$ $\mathrm{CrOOH}),{ }^{33}$ guyanaite $(\beta-\mathrm{CrOOH})^{33}$ and the crystal structure of a chromium(III) dimer compound $^{14}$ as structural models. The relevant paths that were calculated for these model compounds include $\mathrm{Cr}-\mathrm{O}$ and $\mathrm{Cr}-\mathrm{Cr}$ SS interactions, and 3-legged cis- $\mathrm{Cr}-\mathrm{O}-\mathrm{O}$ and 4legged trans- O-Cr-O MS interactions.

To compare the chromium(III) species in the eluates and those on the ion exchange resin, EXAFS spectra of a few resin samples were also collected. For these experiments, the polyethylene column was cut into sections that contained separated chromium(III) oligomers and the sections were sealed and directly mounted on the aluminum sample holder with Scotch tape.

\subsection{Study of oxidation of chromium(III) by hydrogen peroxide in alkaline solutions}

The oxidation kinetics was studied by both conventional UV/Vis absorption spectroscopy and stopped-flow spectroscopy. The growth of the absorbance of chromium(VI) at 372 $\mathrm{nm}$ was monitored. It is known that hydrogen peroxide may decompose through "selfoxidation" to form water and oxygen and the decomposition occurs most rapidly in strongly basic solution. ${ }^{34}$ To minimize this problem, the reaction conditions were 
optimized by preliminary experiments. The initial concentrations of sodium hydroxide and hydrogen peroxide in the majority of the redox experiments were chosen to be below $1.5 \mathrm{~mol} \mathrm{dm}^{-3}$ and $0.5 \mathrm{~mol} \mathrm{dm}^{-3}$, respectively. Under these conditions, no severe selfdecomposition occurred while the pseudo-first-order conditions were still maintained.

3.4.1 Conventional UV/Vis absorption spectroscopy. The experiments were conducted either on a Cary-5G or an Oceanoptics ST 2000 spectrometer. With the latter instrument, the cuvette was maintained inside the glove box under nitrogen atmosphere, and the light was transmitted through optical fibers to and from the cuvette. This setup helped to eliminate the interference by carbon dioxide.

The redox experiments were conducted under the pseudo-first-order conditions with respect to $C_{\mathrm{Cr}}$. As soon as an aliquot of the chromium(III) solution was injected into the cuvette containing appropriate amounts of sodium hydroxide and hydrogen peroxide and rapidly mixed, the absorbance at $372 \mathrm{~nm}$ was monitored as a function of time. Quartz cuvettes with $10 \mathrm{~mm}$ pathlength were used.

In the data processing, the concentration of chromium(III) is expressed as $\left[\mathrm{Cr}_{\mathrm{n}}(\mathrm{III})\right]$ (where $\mathrm{n}=1,2$ and 3 for monomer, dimer and trimer, respectively). A general rate equation is written as

$$
\begin{aligned}
R=-\mathrm{d}\left[\mathrm{Cr}_{\mathrm{n}}(\mathrm{III})\right] / \mathrm{d} t=(1 / \mathrm{n}) \mathrm{d}[\mathrm{Cr}(\mathrm{VI})] / \mathrm{d} t=k\left[\mathrm{Cr}_{\mathrm{n}}(\mathrm{III})\right]^{\mathrm{p}}\left[\mathrm{H}_{2} \mathrm{O}_{2}\right]^{\mathrm{q}}[\mathrm{OH}]^{\mathrm{r}} \\
=k_{\mathrm{OH}}\left[\mathrm{Cr}_{\mathrm{n}}(\mathrm{III})\right]^{\mathrm{p}}\left[\mathrm{H}_{2} \mathrm{O}_{2}\right]^{\mathrm{q}} \\
=k_{\mathrm{obs}}\left[\mathrm{Cr}_{\mathrm{n}}(\mathrm{III})\right]^{\mathrm{p}}
\end{aligned}
$$

At time $\mathrm{t}$,

$$
\begin{aligned}
{\left[\mathrm{Cr}_{\mathrm{n}}(\mathrm{III})\right] } & =\left([\mathrm{Cr}(\mathrm{VI})]_{\infty}-[\mathrm{Cr}(\mathrm{VI})]_{\mathrm{t}}\right) / \mathrm{n} \\
& \propto\left(A_{\infty}-A_{\mathrm{t}}\right) / \mathrm{n}
\end{aligned}
$$


where $[\mathrm{Cr}(\mathrm{VI})]_{\mathrm{t}}$ and $[\mathrm{Cr}(\mathrm{VI})]_{\infty}$ are the concentrations of $\mathrm{Cr}(\mathrm{VI})$ at time $\mathrm{t}$ and at the completion of the reaction, while $A_{\mathrm{t}}$ and $A_{\infty}$ represent the corresponding absorbance of $\mathrm{Cr}(\mathrm{VI})(372 \mathrm{~nm})$ at time $\mathrm{t}$ and at the completion of the reaction, respectively. Control experiments were conducted to determine whether and how rapidly the separated oligomers would change and redistribute after being mixed with sodium hydroxide, so that we could evaluate whether the observed oxidation truly represents the oxidation of the individual chromium(III) species. Aliquots of the chromium(III) monomer, dimer and trimer solutions were injected into sodium hydroxide solutions in the absence of hydrogen peroxide. The absorption spectra were collected at different time intervals, with particular attention paid to the characteristic features in the wavelength region of $300 \mathrm{~nm}$ to $700 \mathrm{~nm}$. No significant changes were observed for chromium(III) dimer and trimer within the time domain of the redox experiments. However, the monomer seemed to change more easily than the dimer or trimer. Subsequent oxidation experiments with the monomer showed that the change of the absorbance at $372 \mathrm{~nm}$ as a function of time could be modeled very well with two exponential components (i.e., two first-order pathways). The slower one was in the same range as the rate of the oxidation of the dimer while the faster one was completed within minutes. We assume that the faster rate component represents the oxidation of the chromium(III) monomer. Using the function of two exponentials and fixing the parameters for the slower component with the results from the oxidation of the dimer, the parameters for the oxidation of the monomer were obtained. To test the validity of this approach of data processing, catch the earlier stages of the oxidation of monomer, and determine the reaction order with respect to the 
monomer more accurately, additional experiments by stopped-flow spectroscopy were conducted.

3.4.2 Stopped-Flow spectroscopy. An Olis (On-Line Instrument Systems, Inc.) U.S.A.-SF attachment to a CARY-14 spectrophotometer at Washington State University was used to study the oxidation of chromium(III) monomer by hydrogen peroxide in alkaline solutions. The stopped-flow spectrometer is a single-beam instrument with two pneumatic plunging syringes that allow rapid mixing of two solutions (mixing time $\sim 1$ millisecond). Typical experiments involved mixing equal volumes of a solution of hydrogen peroxide/sodium hydroxide in one syringe and a solution of chromium(III) monomer in the other syringe. Sodium perchlorate was used to achieve the same ionic strength in both solutions. The formation of chromium(VI) was monitored at $372 \mathrm{~nm}$ with a bandwidth of $1.76 \mathrm{~nm}$ and a pathlength of $20 \mathrm{~mm}$. The voltage readings were converted to absorbance by defining the overall range of voltages: prior to each experiment, the $0 \%$ transmittance was defined by the dark current signal and the $100 \%$ transmittance was defined using a reference solution containing appropriate amounts of sodium hydroxide, sodium perchlorate and hydrogen peroxide.

The initial concentration of chromium(III) monomer in the stopped-flow experiments ranged from $1.2 \times 10^{-5}$ to $1.2 \times 10^{-4} \mathrm{~mol} \mathrm{dm}{ }^{-3}$. The method of "initial rate" was used to process the data. The logarithm plot of the initial rate vs. the concentration of chromium(III) yields the reaction order with respect to chromium. 


\section{Acknowledgments}

This work was supported by the Assistant Secretary for Environmental Management under U.S. Department of Energy Contract No. DE-AC03-76SF0098 at Lawrence Berkeley National Laboratory and Contract No. FG07-98ER14930 at Washington State University. The EXAFS experiments were conducted at SSRL, which is operated by the Department of Energy, Division of Chemical Sciences. The authors thank the colleagues at the Glenn T. Seaborg Center, Lawrence Berkeley National Laboratory, Drs. C. W. Booth and J. Jiang in particular, for the help with the EXAFS experiments and data processing. 


\section{References}

1 Swanson, J. L. Clean Option: An Alternative Strategy for Hanford Tank Waste Remediation. Volume 2: Detailed Description of First Example Flowsheet. PNL8288, Vol.2, Pacific Northwest National Laboratory, Richland, WA, 1993.

2 Baes, C. F. Jr.; Mesmer, R. E. The Hydrolysis of Cations, John Wiley \& Sons, New York, 1976.

3 Postmus, C.; King, E.L. J. Phy. Chem. 1955, 59, 1208.

4 Stiasny, E.; Balanyi, D. Collegium (Darmstadt), 1927, 68.

5 Schaal, R.; Faucherre, J. Bull. Soc. Chim. Fr. 1947, 14, 927.

6 Souchay, P. Bull. Soc. Chim. Fri. 1948, 15, 143.

7 Hall, H.T.; Eyring, H. J. Am. Chem. Soc. 1950, 72, 782.

8 Ardon, M.; Plane, R.A. J. Ame. Chem. Soc. 1959, 81, 3197.

9 Laswick L.A.; Plane, R.A. J. Am. Chem. Soc. 1959, 81, 3564.

10 Finholt, J. E.; Thompson, M. E.; Connick, R. E. Inorg. Chem. 1981, 20, 4151 - 4155.

11 Stunzi, H.; Marty, W. Inorg. Chem. 1983, 22, 2145 - 2150. Stunzi, H.; Rotzinger F. P.; Marty, W. Inorg. Chem. 1984, 23, 2160 - 2164. Rotzinger F. P.; Stunzi, H.; Marty, W. Inorg. Chem. 1986, 25, 489-495. Stunzi, H.; Spiccia, L.; Rotzinger F. P.; Marty, W. Inorg. Chem. 1989, 28, 66 - 71.

12 Kolaczkowski, R.W.; Plane, R.A. Inorg. Chem. 1964, 3, 322.

13 Thompson, M.; Connick, R. E. Inorg. Chem. 1981, 20, 2279 - 2285.

14 Spiccia, L.; Stoeckli-Evans, H.; Marty, W.; Giovanoli, R. Inorg. Chem. 1987, 26, 474 $-482$.

15 Drljaca, A.; Hardie, M. J.; Raston, C. L.; Spiccia, L. Chem. Eur. J. 1999, 5, 22952299.

16 Rai, D.; Sass, M.; Moore, D. A. Inorg. Chem. 1987, 26, 345.

17 Bradley, S. M.; Lehr, C. R.; Kydd, R. A. J. Chem. Soc. Dalton Trans. 1993, 2415 2420.

18 Lindqvist-Reis, P.; Munoz-Paez, A.; Diaz-Moreno, S.; Pattanaik, S.; Persson, I.; Sandstrom, M. Inorg. Chem. 1998, 37, 6675 - 6683.

19 Munoz-Paez, A.; Pappalardo, R. R.; Marcos, E. S. J. Am. Chem. Soc. 1995, 117, 11710-11720. 
20 Bornholdt, K.; Corker, J.M.; Evans, J.; Rummey, J. M. Inorg. Chem. 1991, 30, 1 - 2.

21 Manceau, A.; Charlet, L. J. Colloid \& Interf. Sci., 1992, 148, 425-442, 443- 458.

22 Lumetta, G. J.; Rapko, B. M. Separation Science and Technology, 1999, 34, 14951506.

23 Sylvester, P.; Rutherford, Jr. L. A.; Gonzalez-Martin, A.; Kim, J.; Rapko, B. M.; Lumetta, G. J. Environ. Sci. Technol. 2001, 35, 216-221.

24 Friese, J. I.; Clark, S. B.; personal communication.

25 Zabinsky, S. I.; Rehr, J. J.; Ankudinov, A.; Albers, R. C.; Eller, M. J.; Phys. Rev. B, 1995, 52, 2995.

26 Allen,P .G.; Bucher, J. J.; Clark, D. L.; Edelstein, N. M.; Ekberg, S. A.; Gohdes, J. W.; Hudson, E. A.; Kaltsoyannis, N.; Lukens, W. W.; Neu, M. P.; Palmer, P. D.; Reich, T.; Shuh, D. K.; Tait, C. D.; Zwick, B. D. Inorg. Chem. 1995, 34, 4797.

27 Rai, D.; Hess, N. J.; Rao, L.; Zhang, Z.; Felmy, A. R.; Moore, D. A.; Clark, S. B.; Lumetta, G. J. submitted to J. Sol. Chem.

28 Martell, A. E.; Smith, R. M. Critical Stability Constants, Plenum, New York-London, Vol.3, 1977; First Suppl., 1982; Second Suppl., 1989.

29 Dean, J. A. Analytical Chemistry Handbook, McGraw-Hill, Inc., New York, 1995.

30 Spiccia, L.; Marty, W. Inorg. Chem. 1986, 25, 266 - 271. Spiccia, L.; Marty, W.; Giovanoli, R. Inorg. Chem. 1988, 27, 2660 - 2666.

31 Bucher, J. J.; Edelstein, N. M.; Osborne, K. P.; Shuh, D. K.; Madden, N.; Luke, P.; Pehl, D.; Cork, C.; Malone, D.; Allen, P. G.; SRL '95 Conference Proceedings, Rev. Sci. Instr. 1996, 67, 1.

32 (a) Li, G. G.; Bridges, F.; Booth, C. W. Phys. Rev. 1995, B 52, 6332; (b) Bridges, F.; Booth, C. W.; Li, G. G. Physica B, 1995, 208 \& 209, 12; (c) Booth, C. W.; Ph.D. Thesis, University of California, Santa Cruz, 1996.

33 Christensen, A. N.; Hansen, P.; Lehmann, M.S. J. Solid State Chemistry, 1977, 21, 325-329. Christensen, A. N.; Hansen, P.; Lehmann, M.S. J. Solid State Chemistry, 1976, 19, 299-304.

34 Cotton, F. A.; Wilkinson, G. Advanced Inorganic Chemistry, John Wiley \& Sons, New York, 1988. 
Table 1 Concentration quotients for the oligomerization of chromium(III) in acidic solutions $\left(I=1.0 \mathrm{~mol} \mathrm{dm}^{-3} \mathrm{NaClO}_{4}, 25^{\circ} \mathrm{C}\right)$

$$
\begin{aligned}
p \mathrm{Cr}^{3+} & +q \mathrm{H}_{2} \mathrm{O} \leftrightarrows \mathrm{Cr}_{\mathrm{p}}(\mathrm{OH})_{\mathrm{q}}^{(3 \mathrm{p}-\mathrm{q})+}+q \mathrm{H}^{+} \\
Q_{\mathrm{pq}} & =\left[\mathrm{Cr}_{\mathrm{p}}(\mathrm{OH})_{\mathrm{q}}^{(3 \mathrm{p}-\mathrm{q})+}\right]\left[\mathrm{H}^{+}\right]^{q} /\left[\mathrm{Cr}^{3+}\right]^{p}
\end{aligned}
$$

\begin{tabular}{cccc}
\hline $\log \left(Q_{22} / \mathrm{mol} \mathrm{dm}^{-3}\right)$ & $\log \left(Q_{34} / \mathrm{mol}^{2} \mathrm{dm}^{-6}\right)$ & $\log \left(Q_{46} / \mathrm{mol}^{3} \mathrm{dm}^{-9}\right)$ & Reference \\
\hline-5.5 & -10 & $(-13.5)^{\mathrm{a}}$ & this work \\
-4.82 & -7.94 & & $(2)$ \\
$-5.1^{\mathrm{b}}$ & $-8.3^{\mathrm{b}}$ & & $(10,13)$ \\
-5.34 & $\geq-8.9$ & $\geq-14.1$ & $(11)$ \\
\hline
\end{tabular}

${ }^{a}$ Assuming that the chromium(III) remaining on the column was tetramer.

${ }^{\mathrm{b}}$ Extrapolated from the data for the temperature range $37.5^{\circ} \mathrm{C}-67.5^{\circ} \mathrm{C}$. 
Table 2 EXAFS fitting results for chromium(III) in solution and on resin. The $95 \%$ confidence limit for $R$ and $N: R \pm 0.02 \AA, N \pm 1$ for $\mathrm{Cr}-\mathrm{O}$ and \pm 0.5 for $\mathrm{Cr}-\mathrm{Cr}$. $\sigma$ is the Debye-Waller factor. The amplitude reduction factor, $S_{0}^{2}$, was restrained to be 0.85 , the value found for the octahedral cation $\mathrm{Cr}\left(\mathrm{H}_{2} \mathrm{O}\right)_{6}{ }^{3+}$ as the model for solution chromium(III) species.

\begin{tabular}{|c|c|c|c|c|c|c|c|}
\hline Sample & State & $\begin{array}{c}C_{\mathrm{Cr}} \\
\mathrm{mol} \mathrm{dm}\end{array}$ & $\begin{array}{c}C_{\mathrm{NaOH}} \\
\mathrm{mol} \mathrm{dm}{ }^{-3}\end{array}$ & Interaction & $R, \AA$ & $N$ & $\sigma, \AA$ \\
\hline \multirow[t]{2}{*}{ Monomer } & Solution & 0.01 & $(\mathrm{pH} 2.0)$ & $\mathrm{Cr}-\mathrm{O}^{\mathrm{a}}$ & 1.97 & 5.9 & 0.052 \\
\hline & Resin & & & $\mathrm{Cr}-\mathrm{O}^{\mathrm{a}}$ & 1.97 & 5.8 & 0.048 \\
\hline \multirow[t]{4}{*}{ Dimer } & Solution & 0.001 & (pH 2.8) & $\mathrm{Cr}-\mathrm{O}^{\mathrm{a}}$ & 1.94 & 5.9 & 0.067 \\
\hline & & & & $\mathrm{Cr}-\mathrm{Cr}^{\mathrm{a}}$ & 2.98 & 0.8 & 0.055 \\
\hline & Resin & & & $\mathrm{Cr}-\mathrm{O}^{\mathrm{a}}$ & 1.96 & 5.6 & 0.046 \\
\hline & & & & $\mathrm{Cr}-\mathrm{Cr}^{\mathrm{a}}$ & 2.99 & 1.1 & 0.055 \\
\hline \multirow[t]{2}{*}{ Trimer } & Solution & 0.001 & $(\mathrm{pH} 2.8)$ & $\mathrm{Cr}-\mathrm{O}^{\mathrm{a}}$ & 1.94 & 5.8 & 0.061 \\
\hline & & & & $\mathrm{Cr}-\mathrm{Cr}^{\mathrm{a}}$ & 2.98 & 1.9 & 0.067 \\
\hline \multirow[t]{12}{*}{ Unseparated } & Solution & 0.01 & (pH 3.0) & $\mathrm{Cr}-\mathrm{O}^{\mathrm{a}}$ & 1.98 & 5.7 & 0.056 \\
\hline & & & & $\mathrm{Cr}-\mathrm{Cr}^{\mathrm{a}}$ & 2.98 & 1.5 & 0.062 \\
\hline & & & & $\mathrm{O}-\mathrm{Cr}-\mathrm{O}^{\mathrm{b}}$ & 3.87 & & 0.078 \\
\hline & & 0.01 & 5.5 & $\mathrm{Cr}-\mathrm{O}^{\mathrm{a}}$ & 1.99 & 5.7 & 0.062 \\
\hline & & & & $\mathrm{Cr}-\mathrm{Cr}^{\mathrm{a}}$ & 3.00 & 2.3 & 0.071 \\
\hline & & & & $\mathrm{O}-\mathrm{Cr}-\mathrm{O}^{\mathrm{b}}$ & 3.89 & & 0.081 \\
\hline & & 0.01 & 6.0 & $\mathrm{Cr}-\mathrm{O}^{\mathrm{a}}$ & 1.98 & 5.7 & 0.062 \\
\hline & & & & $\mathrm{Cr}-\mathrm{Cr}^{\mathrm{a}}$ & 2.99 & 2.3 & 0.065 \\
\hline & & & & $\mathrm{O}-\mathrm{Cr}-\mathrm{O}^{\mathrm{b}}$ & 3.89 & & 0.075 \\
\hline & & 0.01 & 7.8 & $\mathrm{Cr}-\mathrm{O}^{\mathrm{a}}$ & 2.00 & 5.6 & 0.056 \\
\hline & & & & $\mathrm{Cr}-\mathrm{Cr}^{\mathrm{a}}$ & 2.99 & 2.7 & 0.065 \\
\hline & & & & $\mathrm{O}-\mathrm{Cr}-\mathrm{O}^{\mathrm{b}}$ & 3.84 & & 0.081 \\
\hline
\end{tabular}

${ }^{\mathrm{a}}$ Single scattering.

${ }^{\mathrm{b}}$ Three 4-legged trans- O-Cr-O multiple scattering interactions, each with a degeneracy of two. 
Table 3. Kinetic data for the oxidation of chromium(III) by hydrogen peroxide $\left(25^{\circ} \mathrm{C}\right)$. The error limits for $k$, and $k$ ' represent the standard deviations at $95 \%$ confidence level (primary kinetic data are provided in Table S1, Supplementary Materials).

\begin{tabular}{cccc}
\hline Chromium Solution & $\begin{array}{c}N_{\mathrm{Cr}-\mathrm{Cr}} \\
\text { at } \sim 2.99 \AA\end{array}$ & $k / \mathrm{min}^{-1}$ & $k / \mathrm{mol} \mathrm{dm}^{-3}$ \\
\hline Monomer & 0 & $327 \pm 6$ & $0.07 \pm 0.09$ \\
Dimer & 0.8 & $11.3 \pm 0.3$ & $0 \pm 0.1$ \\
Trimer & 1.9 & $1.33 \pm 0.04$ & $1.34 \pm 0.04$ \\
Unseparated & 2.7 & $0.032 \pm 0.002$ & $0.53 \pm 0.01$ \\
(in 7.8 mol dm & & & \\
\hline
\end{tabular}


Figure Cations

Fig. 1 Structures of chromium(III) dimer and trimer. (a) Dimer observed in a crystal; ${ }^{14}$ $(b, c)$ Trimer postulated for acidic solution; ${ }^{10,11,13}$ (d) Trimer observed in a crystal. ${ }^{15}$

Fig. 2 Distribution of chromium(III) as a function of $\mathrm{pH}$. The results were obtained by cation exchange with the five solutions described in Experimental Section. $C_{\mathrm{Cr}}=0.05 \mathrm{M}$. Symbols: monomer $(\boldsymbol{O})$, dimer $(\bullet)$, trimer $(\boldsymbol{\Delta})$, unseparated higher oligomers $(\boldsymbol{\square})$.

Fig. $3 k^{3}$-weighted EXAFS (a) and the Fourier transforms (b) of chromium(III) solutions. The phase shift is not corrected in (b). Dashed line: experimental; solid line: fit. All the solutions were filtered with the Gelman $0.2 \mu \mathrm{m}$ nylon filter. The fitting parameters and results are listed in Table 2.

Fig. 4 The initial rate of the oxidation of chromium(III) monomer as a function of the chromium concentration. Data obtained by stopped-flow spectroscopy. $C_{\mathrm{NaOH}}=2.0 \times 10^{-1}$ mol dm${ }^{-3}, C_{\mathrm{H}_{2} \mathrm{O}_{2}}=1.03 \times 10^{-3} \mathrm{~mol} \mathrm{dm}^{-3}, 20 \mathrm{~mm}$ pathlength.

Fig.5 The pseudo first-order rate constant, $k_{\mathrm{obs}}$, as a function of $C_{\mathrm{H}_{2} \mathrm{O}_{2}}$ at constant $C_{\mathrm{NaOH}}$. (a) monomer $\left(C_{\mathrm{Cr}}=3.1 \times 10^{-5}-9.7 \times 10^{-5} \mathrm{~mol} \mathrm{dm}^{-3}\right)$; (b) dimer $\left(C_{\mathrm{Cr}}=7.1 \times 10^{-5}-1.1 \times\right.$ $\left.10^{-4} \mathrm{~mol} \mathrm{dm}{ }^{-3}\right)$; (c) trimer $\left(C_{\mathrm{Cr}}=5.3 \times 10^{-5}-8.1 \times 10^{-5} \mathrm{~mol} \mathrm{dm}{ }^{-3}\right)$. Symbols: $C_{\mathrm{NaOH}}(\mathrm{mol}$ $\left.\mathrm{dm}^{-3}\right)=0.100(\boldsymbol{\bigcirc}, \bigcirc), 0.200(\square), 0.252(\mathbf{\Delta}), 0.504(\boldsymbol{\nabla}), 0.756(+), 1.00(\boldsymbol{\square}), 1.18(\bullet)$. Open and solid symbols represent the data obtained by stopped-flow and conventional spectroscopy, respectively. Detailed conditions of the experiments are provided in Table S1, Supplementary Materials.

Fig. $6 k_{\mathrm{OH}}$ as a function of $C_{\mathrm{NaOH}}{ }^{-1}$. (a) monomer; (b) dimer; (c) trimer. ( $\bigcirc$ ) data from stopped-flow spectroscopy; (O) Data from conventional spectroscopy. Detailed conditions of the experiments are provided in Table S1, Supplementary Materials. 


\section{Supplementary Materials}

Table S1 Kinetic data for the oxidation of chromium(III) by hydrogen peroxide. The error limits for $k_{\mathrm{OH}^{-}}, k$, and $k$ ' represent the standard deviations at $95 \%$ confidence level. Data marked with “*” were obtained by stopped-flow spectroscopy.

Fig. S1 The Fourier transforms of the EXAFS spectra of separated chromium(III) oligomers in solution and on resin. The phase shift is not corrected. Dashed line: experimental; solid line: fit. The fitting parameters and results are listed in Table 2. All the solutions were filtered with the Gelman $0.2 \mu$ m nylon filter.

Fig. S2 The absorbance at $372 \mathrm{~nm}$ as a function of time, observed by stopped-flow spectroscopy. $C_{\mathrm{NaOH}}=2.0 \times 10^{-1} \mathrm{~mol} \mathrm{dm}^{-3}, C_{\mathrm{H}_{2} \mathrm{O}_{2}}=1.03 \times 10^{-3} \mathrm{~mol} \mathrm{dm}^{-3}, 20 \mathrm{~mm}$ pathlength. $C_{\mathrm{Cr}(\mathrm{IIII}), 0}\left(\mathrm{~mol} \mathrm{dm}^{-3}\right)=1.18 \times 10^{-5}$ (I), $2.36 \times 10^{-5}$ (II), $3.77 \times 10^{-5}(\mathrm{III}), 7.04 \times$ $10^{-5}(\mathrm{IV}), 1.18 \times 10^{-4}(\mathrm{~V})$.

Fig. S3 UV/Vis absorption spectra of chromium(III) monomer, dimer, trimer and unseparated solutions. All the solutions were filtered with the Gelman $0.2 \mu \mathrm{m}$ nylon filter. The spectra are shifted with respect to the y-coordinate for better viewing. 